\title{
Ultrasonic Single Element and Sectorized Array Transducers with Omnidirectional 2D Field Distribution for Non-Contact Human-Machine Interface and Echo-Location
}

\author{
Tomas Gomez Alvarez-Arenas ${ }^{1}$, Luis Diez ${ }^{1}$ \\ ${ }^{1}$ Institute of Physical and Information Technologies, Spanish National Research Council (CSIC), \\ Serrano 144, 28006, Madrid, Spain \\ t.gomez@csic.es
}

\begin{abstract}
This work discusses the possibilities of some novel applications for the high frequency $(200 \mathrm{kHz}-500 \mathrm{kHz})$, high sensitivity (-20 dB- $-35 \mathrm{~dB})$ and wide band (> $50 \% @-20 \mathrm{~dB})$ air-coupled transducers that have been used so far for nondestructive testing and materials characterization at CSIC. These applications refer to non-contact human-machine interfaces, gesture recognition and echolocation. Compared with other technologies, the relatively higher frequency and bandwidth permit to achieve better resolution and the higher sensitivity permit to reach longer distances. In addition, this later feature also permits to reduce the number of transducers. This is achieved by using a conical reflector that provides an omnidirectional (2D) acoustic field. Examples of applications and realizations are shown by using single element $250 \mathrm{kHz}$ transducers; and 8-elements $400 \mathrm{kHz}$ sectorized array transducers. This later type of air-coupled array is introduced here for the first time.
\end{abstract}

Index Terms-Piezoelectric transducers; ultrasonic transducer arrays; impedance matching; robot sensing systems; sensor systems and applications.

\section{INTRODUCTION}

There is a technology for bridging the human-computer barrier, a revision of different technologies can be seen in [1]. Air-coupled ultrasound is a very interesting technology for non-contact human machine interfaces and gesture recognition systems. These systems are of interest in different fields, the best example is provided by the gaming industry, yet there are also some other fields where avoiding contact between the user and the machine (computer, smartphone, etc.) may present an advantage. Examples appear in different robotic applications, in the use of machines and interfaces by medical doctors during different medical procedures, etc. Technologies based in laser and image processing have been widely used and there are also some applications based in air-coupled ultrasound [2]-[5]. In this case, the main problems are to find efficient ultrasonic transmitter/receivers, to reduce the number of

Manuscript received 19 December, 2016; accepted 21 May, 2017

This research was funded by grant (DPI2016-78876-R-AEI/FEDER, UE) from the Spanish State Research Agency (AEI) and the European Regional Development Fund (ERDF / FEDER). transmitters/receivers and to achieve good spatial accuracy over the whole volume of the space of human-machine interaction for the application under consideration. The selection of the technology to be used largely depends on the minimum and maximum distances, the volume of interaction, the size of the object to be detected (the tip of a pen, a finger, a hand a head or a whole human body), its movement, the required accuracy and other restrictions in terms of size, volume, weight and consume of the device.

\section{TRANSDUCERS AND ELECTRONIC EQUIPMENT}

Two different types of air-coupled transducers have been employed in this work, all of them designed and fabricated at CSIC: single element transducers and sectorized array transducers [6]-[9]. The key design elements are: the centre frequency and the bandwidth (relatively high to achieve a good spatial resolution) and the sensitivity (high enough so that it is possible to generate a $2 \mathrm{D}$ omnidirectional field and detect small objects at large distances). All transducers have been driven by using a Panametrics pulser-receiver, either the 5077 or the 5058 , the former provides a semicycle of square wave that can be tuned to the transducer centre frequency, with amplitude between $100 \mathrm{~V}$ and $400 \mathrm{~V}$; while the later provides a wide band spike excitation with peak amplitude in the range $50 \mathrm{~V}-900 \mathrm{~V}$. Display and digitalization of the received echoes were performed by a DPO 5054 Tektronix oscilloscope.

In order to compare the obtained results with those that can be obtained by using commercial air-coupled ultrasonic transducers, two other transducers were also used: the $40 \mathrm{kHz}$ Murata transducer (MA 40S4S) and the $200 \mathrm{kHz}$ Multicomp transducer (MCUSD19A200B11RS).

\section{A. Single Element $250 \mathrm{kHz}$ Transducers}

The single element $250 \mathrm{kHz}$ transducers were fabricated using a 1-3 connectivity piezoelectric composite disk (65\% volume fraction of PZT5A in epoxy resin matrix), $25 \mathrm{~mm}$ diameter, poled in the thickness direction and with the first thickness mode at $250 \mathrm{kHz}$. Matching to the air is achieved by a layered impedance matching layer made of a stack of five sublayers attached to the radiating surface. Peak 
sensitivity is $-25 \mathrm{~dB}$ at $290 \mathrm{kHz}$ and $-20 \mathrm{~dB}$ bandwidth is $46 \%$. Impulse response and sensitivity vs frequency of these transducers can be seen in [6].

\section{B. Sectorized 8-Elements $450 \mathrm{kHz}$ Transducers}

The sectorized 8 elements $450 \mathrm{kHz}$ transducer is introduced for the first time in this work. It was fabricated using a 1-3 connectivity piezoelectric composite disk similar to the ones used before but with the first thickness mode located at $400 \mathrm{kHz}$. Matching to the air is achieved by following a similar procedure. The sectorized array is kerfless, hence, the isolation between the different elements is obtained by removing the metallization along the edges of the sector elements only on the back surface, while the metallization in the front surface is continuous and ground connected, see Fig. 1. All 8 elements are identical. Figure 2 shows the response of one element of the sectorized array in pulse-echo mode with a flat reflector at $30 \mathrm{~mm}$.

Figure 3 shows a picture of some of the monolithic $250 \mathrm{kHz}$ air coupled transducers and the $450 \mathrm{kHz}$ sectorized 8 -elements transducer.

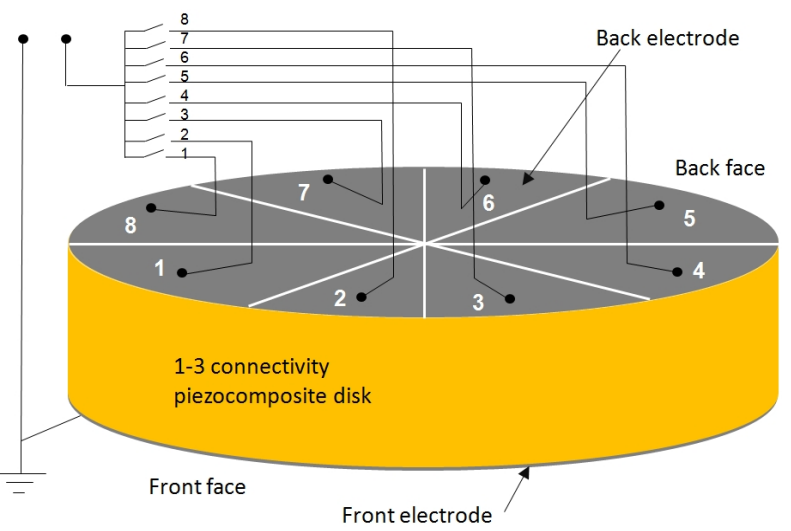

Fig. 1. Schematic representation of the sectorized array disk.

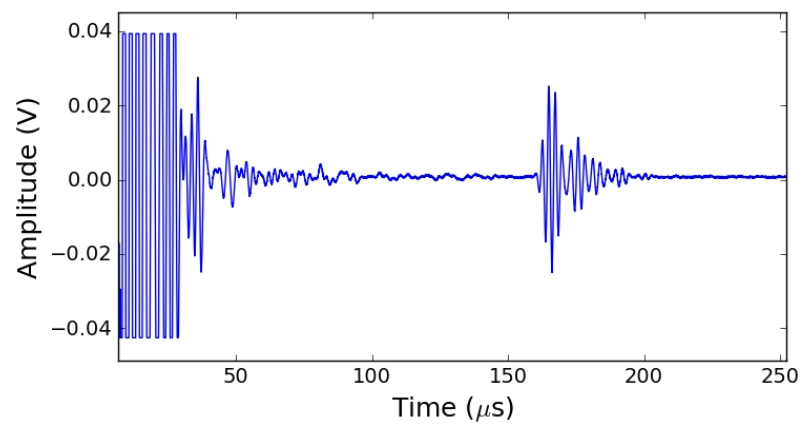

a)

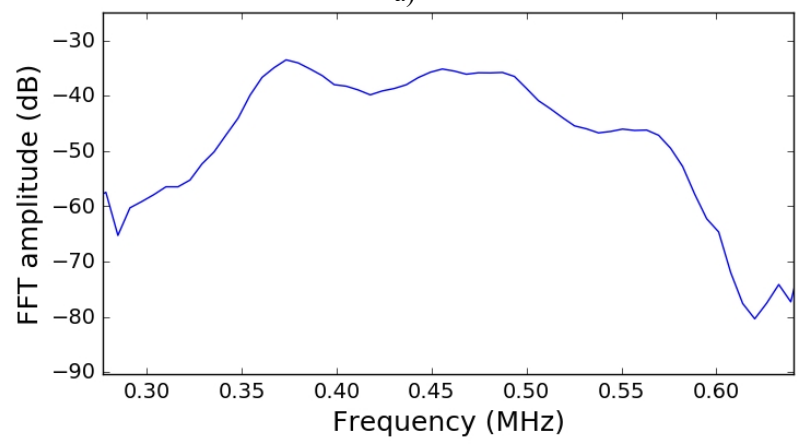

b)

Fig. 2. Response of the element \#1 of the sectorized array in pulse echo mode using the Panametrics 5077 (100 V, $0 \mathrm{~dB})$. Reflector at $30 \mathrm{~mm}$.

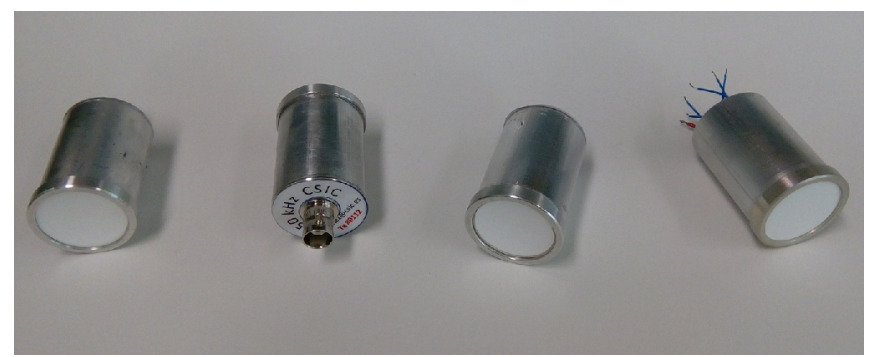

Fig. 3. Picture of the $250 \mathrm{kHz}$ single element air coupled transducers (three transducers on the left) with BNC connector and the 8 elements $400 \mathrm{kHz}$ sectorized array transducer (right) without any connector.

\section{Conical Reflector (or Field Spreader) to Generate A 2D Omnidirectional Field}

The role of the conical reflector is to spread the acoustic field so that the number of transmitters/receivers can be minimized and the potential volume of interaction maximized. Two main configurations have been used: i) cantered conical reflector, to achieve a $360^{\circ}$ acoustic field distribution and ii) Laterally displaced conical reflector to achieve a $180^{\circ}$ acoustic field distribution. These two configurations are shown in Fig. 4.

Figure 5 (right) shows the reduction of the sensitivity of the transducers due to the use of the conical reflectors. Case 1 is obtained for two $250 \mathrm{kHz}$ transducer in pitch-catch mode without conical reflector, while cases 2 and 3 represent the $250 \mathrm{kHz}$ transducers with conical reflectors $\left(180^{\circ}\right.$ and $360^{\circ}$ configurations, respectively). For comparison purposes the SNS band of the Murata and the Multicomp transducers without conical reflector is also shown. The different configurations of transducers and conical reflectors used for these measurements is also shown on Fig. 5 (left).

Figure 6 shows the experimental set-up for through transmission measurements using two transducers (one transmitter, $\mathrm{Tx}$, and one receiver, $\mathrm{Rx}$ ) with two conical reflectors in the $180^{\circ}$ configuration and the received signal measured using the $250 \mathrm{kHz}$ and the $40 \mathrm{kHz}$ transducers.

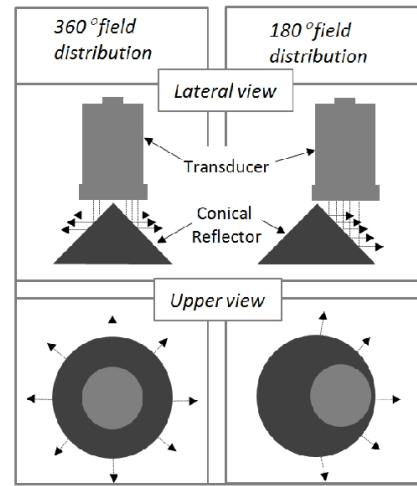

a)

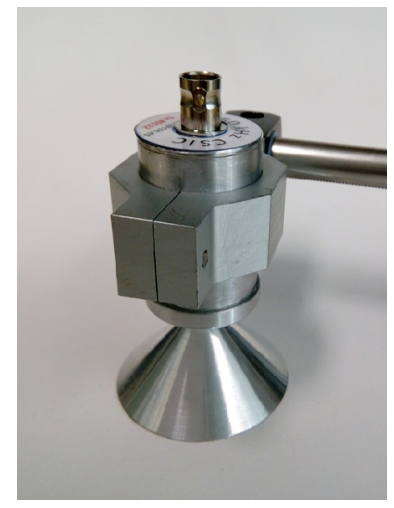

b)
Fig. 4. Schematic representation of the two configurations employed for the conical reflector (a). $250 \mathrm{kHz}$ transducer with the conical reflector in the $360^{\circ}$ field distribution configuration (b).

Figure 7(a) shows the directivity pattern of the sectorized array with the conical reflector in the $360^{\circ}$ configuration obtained by operating each element of the array in pulseecho mode (using the $5077 \mathrm{PR}$ ) and measuring the peak to peak amplitude of the echo originated by a $20 \mathrm{~mm}$ cylindrical reflector located at $200 \mathrm{~mm}$ away from the 
transducer/conical reflector axis. Figure 7(b) and Fig. 7(c) show a schematic representation of the experimental set-up.
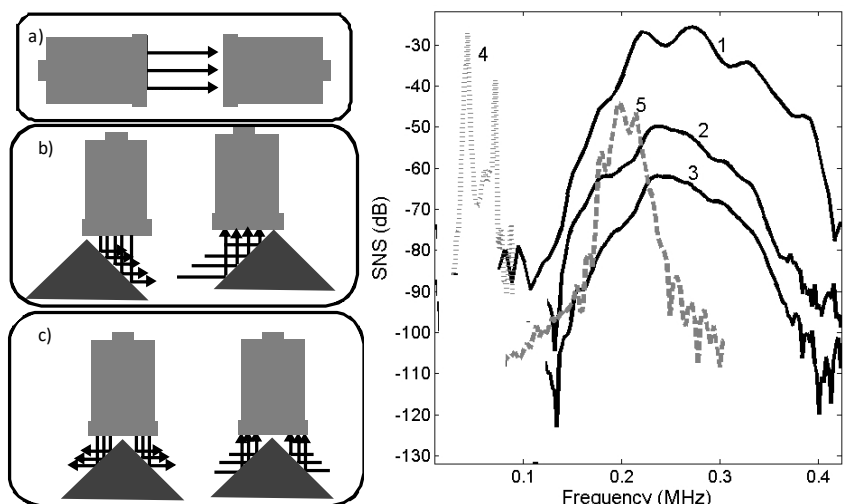

Fig. 5. Comparison of the sensitivity (SNS) frequency band. Left: representation of the different configurations employed. Right: SNS vs frequency for 1: $250 \mathrm{kHz}$ transducers with no conical reflector (a). 2: Same as 1$)$, but using conical reflectors $\left(160^{\circ}\right.$ configuration, b), 3: Same as 1$)$ but using conical reflectors $\left(360^{\circ}\right.$ configuration, c), 4: $40 \mathrm{kHz}$ Murata transducer with no conical reflector (a). 5) Multicomp $200 \mathrm{kHz}$ transducer with no conical reflector (a).
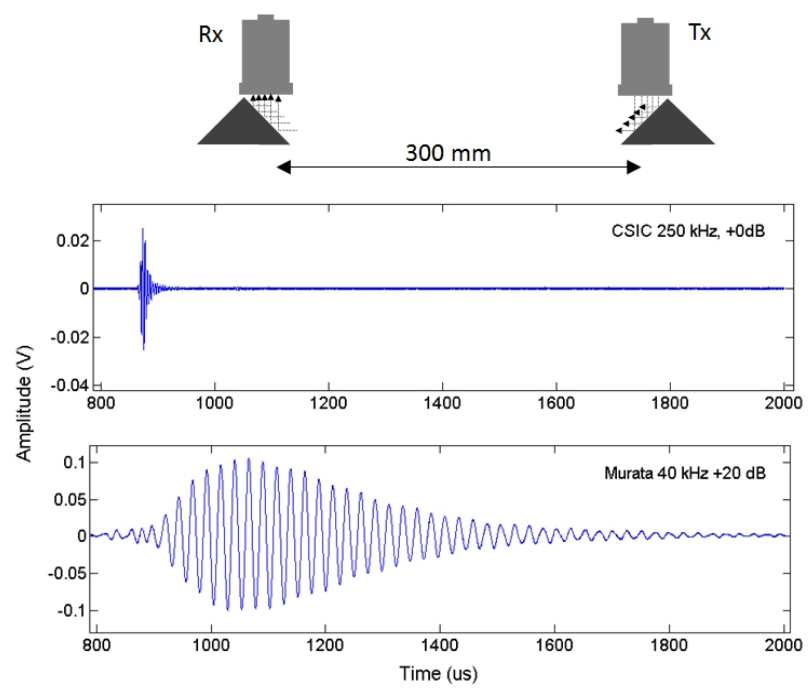

Fig. 6. Comparison of received signals using $250 \mathrm{kHz}$ single element transducer and Murata $40 \mathrm{kHz}$ transducers with conical reflectors $180^{\circ}$ configuration and in through transmission (shown above the figure).

a)

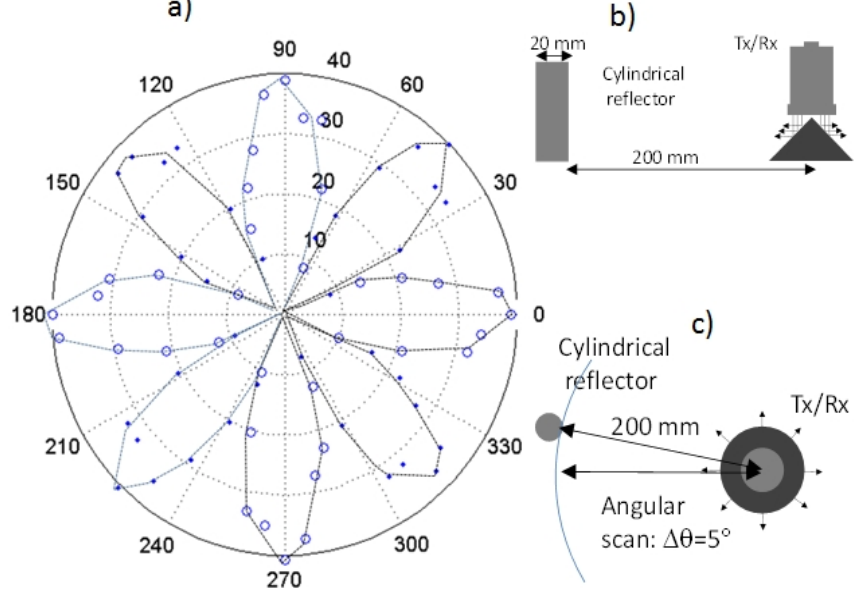

Fig. 7. Directivity pattern of the 8 elements sectorized $400 \mathrm{kHz}$ array measured with the conical reflector in the $360^{\circ}$ configuration and in pulseecho mode using a cylindrical reflector $(20 \mathrm{~mm}$ diameter) located at 200 $\mathrm{mm}$ distance and rotated $360^{\circ}$ around the transducers in steps of $5^{\circ}$ (a). and c) lateral and upper view of the experimental configuration (b).

\section{TRANSDUCERS CONFIGURATION FOR 2D ECHOLOCATION}

\section{A. Two Single Element Transducers in Pulse-echo Operation Mode}

Figure 8 shows the configuration to determine the location of an object (cylindrical reflector) in a half space using two transducers with conical reflectors in $180^{\circ}$ configuration, both transducers operate in pulse-echo mode. Two time of flights (tof) between transducer and reflector are measured $\left(t o f_{1}\right.$ and $\left.t o f_{2}\right)$ and hence two distances are obtained $\left(\mathrm{D}_{1}\right.$ and $\mathrm{D}_{2}$ ). The position of the object is worked out as the intersection of the two circumferences having radii equal to $\mathrm{D}_{1}$ and $\mathrm{D}_{2}$ and centred at $\mathrm{Tr} / \mathrm{Rx} 1$ and $\mathrm{Tx} / \mathrm{Rx} 2$, respectively.

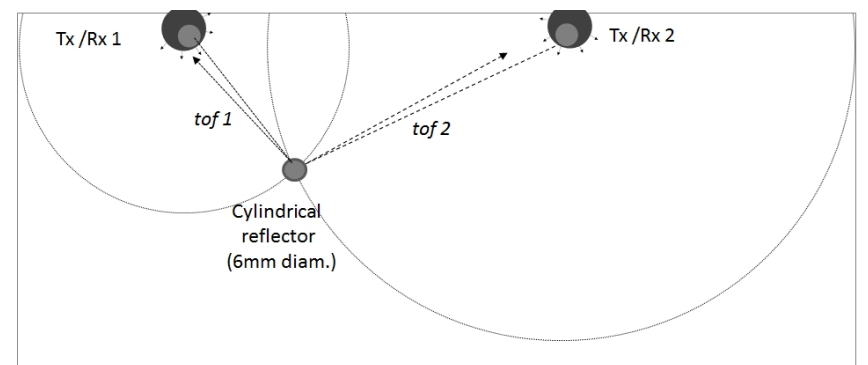

Fig. 8. Schematic representation of the transducer configuration for echolocation of a cylindrical object using two transducers with conical reflector in $180^{\circ}$ configuration.

\section{B. Three Single Element Transducers in Pitch-catch Mode: One Transmitter (Tx) and Two Receivers (Rx)}

Fig. 9 shows the configuration to determine the location of an object (cylindrical reflector) in a half space using three transducers with conical reflectors in $180^{\circ}$ configuration. In this case there is only one transmitter and the two receivers. Two time of flights (tof) between the transmitter and the two receivers are measured $\left(t o f_{1}\right.$ and $t o f_{2}$ ) and hence two distances are obtained $\left(\mathrm{D}_{1}\right.$ and $\left.\mathrm{D}_{2}\right)$. The position of the object is worked out as the intersection of the two ellipses whose foci are located at the transducers positions, that is, $\mathrm{Tx}$ and $\mathrm{Rx} 1$ for one ellipse and Tx and $\mathrm{Rx} 2$ for the other.

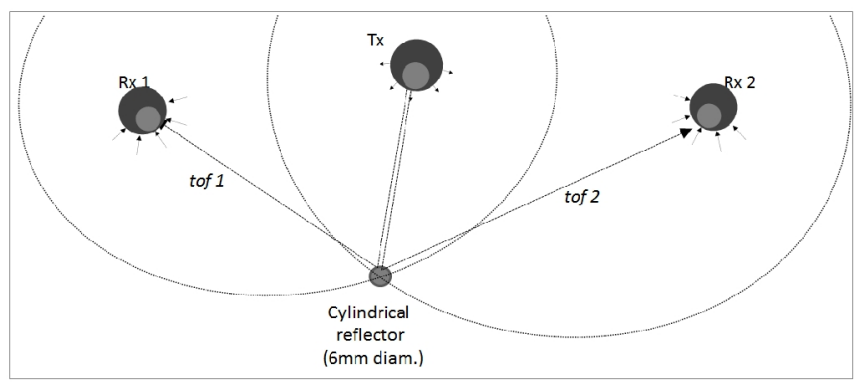

Fig. 9. Schematic representation of the transducer configuration for echolocation of a cylindrical object using three transducers with conical reflector in $180^{\circ}$ configuration.

\section{One Sectorized Array Transducer in Pulse-echo Mode} and Conical Reflector in $360^{\circ}$ Configuration

Figure 10 shows the configuration using one sectorized array transducer with a conical reflector in $360^{\circ}$ configuration. Location of objects is directly determined from tof (distance) and the number of the element that received the echo (direction). 


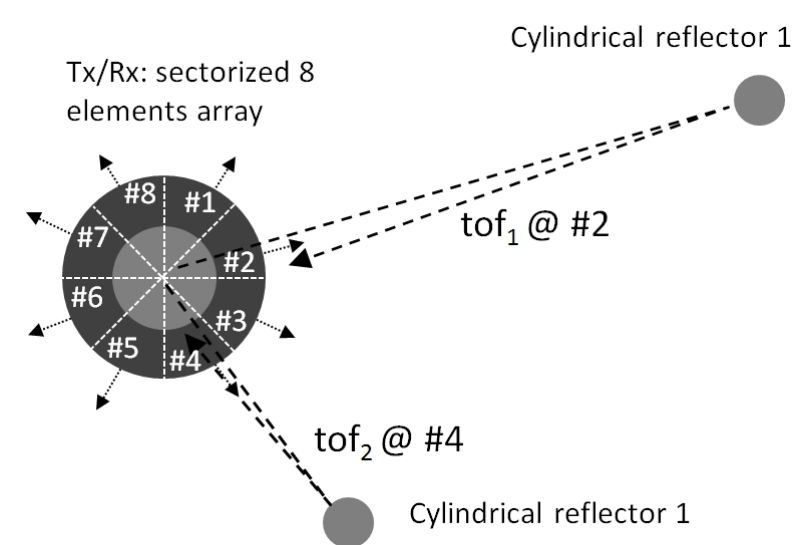

Fig. 10. Schematic representation of the transducer configuration for echolocation of a cylindrical object using one sectorized array transducer with a conical reflector in $360^{\circ}$ configuration.

\section{RESULTS}

Figure 11 and Fig. 12 show the received echo from flat and cylindrical obstacles, using single element $250 \mathrm{kHz}$ and $40 \mathrm{kHz}$ Murata transducers in pitch-catch mode and with conical reflectors in $180^{\circ}$ configuration. It can be clearly seen that the $40 \mathrm{kHz}$ transducers can hardly distinguish the echo from the obstacle from the direct transmission from $\mathrm{Tx}$ to $\mathrm{Rx}$. This problem does not appear in the $250 \mathrm{kHz}$ transducer thanks to the higher centre frequency and wider band. In addition, signal amplitude is higher for the $250 \mathrm{kHz}$ in spite of the larger attenuation in the air.

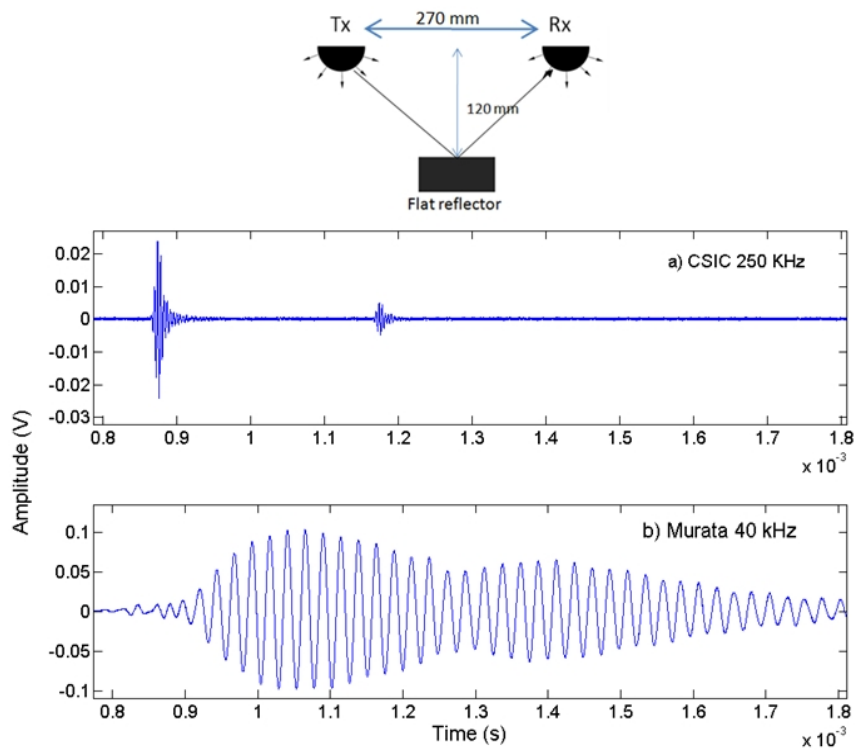

Fig. 11. Received echo from a flat obstacle located at $120 \mathrm{~cm}$ from the transducers using: a) two $250 \mathrm{kHz}$ transducers element and b) two $40 \mathrm{kHz}$ Murata transducers with a conical reflector in $180^{\circ}$ configuration. The signal at $\sim 0.85 \mathrm{~ms}$ is the direct transmission from $\mathrm{Tx}$ to $\mathrm{Rx}$ while the signal at $\sim 1.15 \mathrm{~ms}$ correspond to the echo from the cylindrical reflector/obstacle.

Figure 13 shows the received echo from flat and cylindrical (45 mm diam.) obstacles located at $20 \mathrm{~cm}$ from the $450 \mathrm{kHz}$ sectorized array transducer, using one element, with a conical reflector $\left(360^{\circ}\right.$ configuration). It can be seen that the amplitude and SNR of the echo obtained from the flat obstacle is larger, but that signal shapes is almost the same.

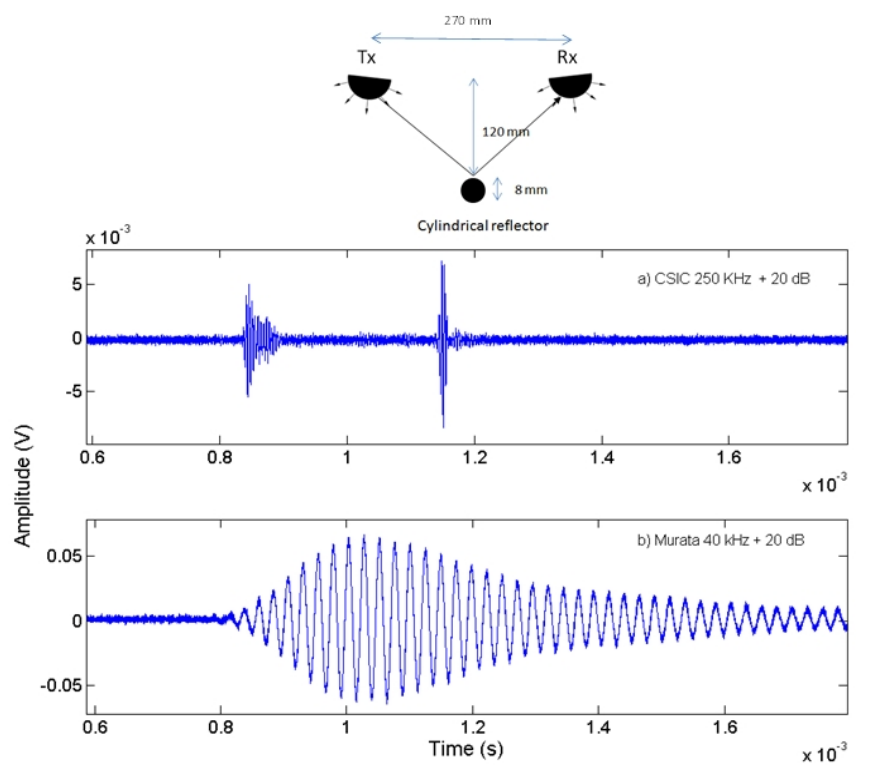

Fig. 12. Received echo from a cylindrical obstacle located at $120 \mathrm{~cm}$ from the transducers with a conical reflector in $180^{\circ}$ configuration using: a) two $250 \mathrm{kHz}$ transducers element and b) two $40 \mathrm{kHz}$ Murata transducers. Signal at $\sim 0.85 \mathrm{~ms}$ is the direct transmission from Tx to Rx. Signal at $\sim 1.15 \mathrm{~ms}$ correspond to the echo from the cylindrical reflector/obstacle.

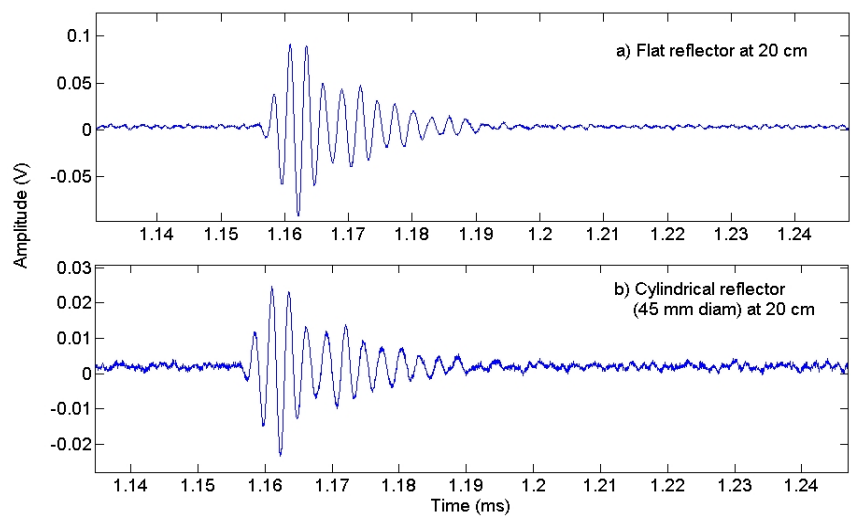

Fig. 13. Received echo from a flat and a cylindrical (45 $\mathrm{mm}$ diameter) obstacle located at $20 \mathrm{~cm}$ from the transducer using element \#1 of the sectorized array with a conical reflector in $360^{\circ}$ configuration.

\section{CONCLUSIONS}

This preliminary work analyses the possibility of using air-coupled ultrasonic transducers in the frequency range $200 \mathrm{kHz}-500 \mathrm{kHz}$ for precise echolocation that could be further applied to gesture recognition and non-contact human-machine interfaces. The key elements are the extremely high sensitivity (between -19 and $-30 \mathrm{~dB}$ ) and the relatively high centre frequency (between $200 \mathrm{kHz}$ and $500 \mathrm{kHz}$ ) and wide band response (>50\% at $-20 \mathrm{~dB}$ ). These features make possible to obtain a much better resolution that that obtained with conventional lower frequency transducers $(40 \mathrm{kHz})$, making possible to resolve a reflector at a close distance $(<120 \mathrm{~mm})$, as well as small spherical reflectors ( $8 \mathrm{~mm}$ diam.). In addition, the very high sensitivity makes possible the use of conical reflectors to spread the beam in either $180^{\circ}$ or $360^{\circ}$ directions, while keeping a reasonable SNR and sensitivity (between $-30 \mathrm{~dB}$ and $40 \mathrm{~dB}$ ). This makes possible to reduce the number of transducer required. An alternative, based on a novel 
sectorized air-coupled array, presented here for the first time, has been also shown. Its response is similar to that of the single element counterparts, but these arrays enable a reduction of the number of transducers required.

\section{REFERENCES}

[1] S. S. Rautaray, A. Agrawal, "Vision based hand gesture recognition for humancomputer interaction: a survery", Artificial Inteligent Review, vol. 43, no. 1, pp. 1-54, 2015. [Online]. Available: https://doi.org/10.1007/s10462-012-9356-9

[2] T. Chiu, H. Deng, S. Chang, "Implementation of ultrasonic touchless interactive panel using the polymer-based CMUT array", in IEEE Sensors 2009, 2009, pp. 625-630.

[3] K. K. Park, B. T. Khuri-Yakub, "3-D airborne ultrasound synthetic aperture imaging based on capacitive micromachined ultrasonic transducers", Ultrasonics, vol. 53, no. 7, pp. 1355-62, 2013. [Online]. Available: https://doi.org/10.1016/j.ultras.2013.04.003

[4] K. Kalgaonkar, B. Raj, "One-handed gesture recognition using ultrasonic Doppler sonar", IEEE Int. Conf. Acoust. Speech Signal
Process. (ICASSP 2009), 2009, pp. 1889-1892. [Online]. Available: https://doi.org/10.1109/ICASSP.2009.4959977

[5] R. Li, T-W Lee, H. Nelson, S. K. Gupta, "Touchless sensing and gesture recognition using continuous wave ultrasound signals", USA Patent, 2012/0001875 A1, 2012.

[6] T. E. Gomez Alvarez-Arenas, "Air-coupled piezoelectric transducers". [Online]. Available: https://us-biomat.com/research/aircoupled-ultrasound-transducers/

[7] T. E. Gomez Alvarez-Arenas, "Acoustic impedance matching of piezoelectric transducers to the air", IEEE Trans. Ultrason. Ferroelectr. Freq. Control, vol. 51, no. 5, pp. 624-33, 2004. [Online]. Available: https://doi.org/10.1109/TUFFC.2004.1308697

[8] S. P. Kelly, G. Hayward, T. E. G. Alvarez-Arenas, "Characterization and assessment of an integrated matching layer for air-coupled ultrasonic applications", IEEE Trans. Ultrason. Ferroelectr. Freq. Control, vol. 51, no. 10, pp. 1314-23, 2004. [Online]. Available: https://doi.org/10.1109/TUFFC.2004.1350960

[9] T. E. Gomez Alvarez-Arenas, T. Shrout, S. J. Zhang, H. Lee, “Aircoupled transducers based on 1-3 Connectivity single crystal piezocomposites", in IEEE International Ultrasonics Symposium, 2012, pp. 2230-2233. [Online]. Available: https://doi.org/10.1109/ ULTSYM.2012.0557 